\title{
Novel method for dynamic control of intracranial pressure
}

\author{
Mark G. Luciano, MD, PhD, ${ }^{1,3}$ Stephen M. Dombrowski, PhD, ${ }^{1}$ Sara Qvarlander, PhD, ${ }^{1,2}$ \\ Serge El-Khoury, MS, ${ }^{1}$ Jun Yang, MD, MS, ${ }^{1}$ Suraj Thyagaraj, MS, ${ }^{4,5}$ and Francis Loth, $\mathrm{PhD}^{4}$
}

\begin{abstract}
'Department of Neurosurgery, Section of Pediatric Neurosurgery, Neurological Institute, Cleveland Clinic, Cleveland, Ohio; 2Department of Radiation Sciences-Biomedical Engineering, Umeå University, Umeå, Sweden; ${ }^{3}$ Department of Neurosurgery, Johns Hopkins University, Baltimore, Maryland; and ${ }^{4}$ Department of Mechanical Engineering, ${ }^{5}$ Conquer Chiari Research Center, Department of Mechanical Engineering, The University of Akron, Ohio
\end{abstract}

\begin{abstract}
OBJECTIVE Intracranial pressure (ICP) pulsations are generally considered a passive result of the pulsatility of blood flow. Active experimental modification of ICP pulsations would allow investigation of potential active effects on blood and CSF flow and potentially create a new platform for the treatment of acute and chronic low blood flow states as well as a method of CSF substance clearance and delivery. This study presents a novel method and device for altering the ICP waveform via cardiac-gated volume changes.
\end{abstract}

METHODS The novel device used in this experiment (named Cadence) consists of a small air-filled inelastic balloon (approximately $1.0 \mathrm{ml}$ ) implanted into the intracranial space and connected to an external programmable pump, triggered by an R-wave detector. Balloons were implanted into the epidural space above 1 of the hemispheres of 19 canines for up to 10 hours. When activated, the balloons were programed to cyclically inflate with the cardiac cycle with variable delay, phase, and volume. The ICP response was measured in both hemispheres. Additionally, cerebral blood flow (heat diffusion and laser Doppler) was studied in 16 canines.

RESULTS This system, depending on the inflation pattern of the balloon, allowed a flattening of the ICP waveform, increase in the ICP waveform amplitude, or phase shift of the wave. This occurred with small mean ICP changes, typically around $\pm 2 \mathrm{~mm} \mathrm{Hg}(15 \%)$. Bilateral ICP effects were observed with activation of the device: balloon inflation at each systole increased the systolic ICP pulse (up to $16 \mathrm{~mm} \mathrm{Hg}, 1200 \%$ ) and deflation at systole decreased or even inverted the systolic ICP pulse $(-0.5$ to $-19 \mathrm{~mm} \mathrm{Hg},-5 \%$ to $-1600 \%)$ in a dose-(balloon volume) dependent fashion. No aphysiological or deleterious effects on systemic pressure $(\leq \pm 10 \mathrm{~mm} \mathrm{Hg} ; 13 \%$ change in mean pressure) or cardiac rate $(\leq \pm$ 17 beats per minute; $16 \%$ change) were observed during up to 4 hours of balloon activity.

CONCLUSIONS The results of these initial studies using an intracranially implanted, cardiac-gated, volume-oscillating balloon suggest the Cadence device can be used to modify ICP pulsations, without physiologically deleterious effects on mean ICP, systemic vascular effects, or brain injury. This device and technique may be used to study the role of ICP pulsatility in intracranial hemo- and hydrodynamic processes and introduces the creation of a potential platform of a cardiac-gated system for treatment of acute and chronic low blood flow states, and diseases requiring augmentation of CSF substance clearance or delivery.

https://thejns.org/doi/abs/10.3171/2016.4.JNS152457

KEY WORDS intracranial pressure; cerebral blood flow; cerebrospinal fluid; implantable device; pulsatility; diagnostic and operative techniques

$\mathrm{T}$ HE intracranial pressure (ICP) waveform may be considered largely the passive result of cardiacinduced pulsations of incoming blood. However, within the rigid cranium, the potential effect of these pressure waves acting on brain morphology, blood flow, and CSF motion is not well understood. The ability to indepen- dently alter the ICP waveform magnitude, shape, and/or phase allows for investigation of the role of ICP pulsation on these physiological processes. A method and device for altering the ICP waveform through induced cardiacgated changes in effective intracranial volume is herein described. These alterations may allow for both a better

ABBREVIATIONS $\triangle \mathrm{BV}=$ (theoretical) amplitude of the balloon volume variation; $\mathrm{ABP}=$ arterial blood pressure; $\mathrm{A} / \mathrm{D}=$ analog to digital translating; $\mathrm{CBF}=\mathrm{cerebral}$ blood flow; $\mathrm{CBF}_{L \mathrm{D}}=\mathrm{CBF}$ laser Doppler; $\mathrm{CBF}_{\mathrm{TD}}=\mathrm{CBF}$ thermal diffusion; $\mathrm{CBF}_{\mathrm{V}}=\mathrm{CBF}$ velocity; $\mathrm{EKG}=$ electrocardiogram; ICP = intracranial pressure; $\mathrm{TPU}=$ tissue perfusion unit. SUBMITTED October 23, 2015. ACCEPTED April 6, 2016.

INCLUDE WHEN CITING Published online July 15, 2016; DOI: 10.3171/2016.4.JNS152457. 
understanding of intracranial dynamics and development of new modalities of clinical treatment. These clinical situations include acute and chronic low blood flow states such as stroke and dementia. In addition, because CSF solute distribution may be altered by CSF pulsatility, ${ }^{11,14}$ this method may be studied in clinical settings requiring enhanced clearance or substances delivery, such as neurodegenerative disease and tumors.

The concept of intracranial pulsatility encompasses the cardiac-induced variations in flow and pressure for both blood and CSF. These dynamics have long been considered significant in brain physiology and pathophysiology, including areas related to stroke, aging, and neurodegenerative diseases. ${ }^{1,15,18}$ Both positive and negative effects of the incoming cerebral blood flow (CBF) pulsatility have been suggested, but are still debated., ${ }^{1,21,24}$ While some arterial pulsatility may increase flow efficiency, ${ }^{12}$ increased blood flow pulsatility may also be undesirable. For example, increased blood flow pulsatility delivered to the brain capillaries via aging atherosclerotic arteries may result in "pulse wave encephalopathy," a potential contributor to brain aging and dementia. ${ }^{1,2}$ A role for CSF flow and pressure pulsation has been suggested in the pathophysiology of various forms of hydrocephalus, cysts, brain malformation, and CSF clearance and absorption has also been suggested. ${ }^{3,6,9,14,20,22,26}$

The closed cranium, with regulated inflow and outflow, clearly links together any changes in pressure with the flow of blood, interstitial fluid, and CSF. Unlike other unrestricted organs, where systolic expansion of the inflowing vessels and parenchyma is less restricted, any systolic vessel expansion must be accommodated immediately by increasing the exit of venous blood and CSF in the cranium..$^{10,12,19,25,27}$ Furthermore, the pulse-smoothing Windkessel effect, $7,8,28$ which is essentially a temporary systolic increase in arterial storage volume, is hampered due to the constraints of a rigid cranium. The brain with its tightly regulated high blood flow may be uniquely vulnerable to cardiac-related pulsatile stress due to the constraint of the rigid cranium, which restricts the Windkessel mechanism. In addition, with aging, rigid atherosclerotic peripheral arteries may result in a weakening of the systemic Windkessel mechanism delivering greater pulsation to brain capillaries. ${ }^{18}$

In this study we suggest placing a small inelastic balloon or bladder in, or adjacent to, a cranial or spinal CSF space epidurally, subarachnoidally, or intraventricularly, such that control of the balloon volume would directly alter CSF space volume and pressure, to affect global ICP. If the balloon expansion and contraction is gated to the cardiac cycle, the ICP waveform shape and phase can be, in theory, manipulated. Because the oscillation (i.e., balloon inflation/deflation) would be symmetrical over the cardiac cycle, the overall ICP mean may not be significantly affected. The ability to independently alter the ICP waveform with an implantable cardiac-gated oscillating device is the central purpose of the method and device investigated here (the Cadence system; US patent nos. 8,956,379 and $9,011,378) .{ }^{16,17}$

The concept of an inflatable intracranial balloon is not itself unprecedented. A previous experimental report by
DiRocco et al. described a balloon experimentally placed in the lateral CSF ventricle and timed to expand during systole in an attempt to demonstrate a mechanism of ventricular expansion., ${ }^{4,523}$ The present system was developed for more diversity in shape, location, timing, and waveform of volume change, and for the potential alteration of various physiological systems. With different cardiac-gated balloon volume waveforms, the system can augment, reduce, or reverse the normal ICP waveform. In addition, the implantable device may be envisioned in various embodiments, from a wide thin bladder in the cranial epidural space to a cylindrical addition to a shunt's ventricular drainage catheter. As a result, the Cadence system may enable study of the effect of a variety of ICP pulse wave modifications on all 3 major intracranial constituents: CSF, CBF and cerebral blood pressure, and brain morphology.

This paper describes the design and implementation of the Cadence device, and reviews some preliminary results from canine experiments that demonstrate potential effects and uses of the system. Further quantitative and comparative studies will be required to evaluate its potential impact. Although the location, shape, and behavior of the Cadence system may be specifically varied, the initial studies described here focus largely on a cranial epidural approach and a flat design of the balloons. General effectiveness of the Cadence device is discussed with regard to: 1) altering the ICP waveform, 2) effects on systemic physiology and on the adjacent brain, and 3) alterations of CBF. This study seeks to prove the concept that the cardiacgated system can alter the ICP waveform and furthermore have an effect on CBF. Preliminary data on histological effects of the cranially implanted balloon and Cadence activation are also described.

\section{Methods}

\section{Cadence System}

The Cadence system is an implantable balloon with a shape and size that allows for small (approximately 1.0 $\mathrm{ml}$ ), inelastic, nontraumatic inflation and deflation gated to the cardiac cycle. It consists of an implantable balloon connected via catheter to a reversible external pump, with a stepper motor triggered by an R-wave detector and computer-controlled with custom-designed software for waveform programming (Fig. 1). The oscillating piston pump used here is a benchtop device, although minimized and physiologically powered device versions may be considered for future applications. Similarly, the bladders used here are designed broad and flat for convexity placement, but they may also be tailored to cisternal, ventricular, or lumbar placement. The common principle is that the oscillating volume of the balloon changes the effective volume of the CSF spaces it occupies or influences. Schematics of different balloon shapes, dimensions, and locations are shown in Fig. 2.

\section{Implanted Balloon}

The prototype balloons were made from a flexible, inelastic, biocompatible silicone (Cleveland Clinic Polymer Lab) and designed for intracranial insertion into the epidural space via bur hole. The balloons were connected to 


\section{Cadence system}

\section{Experimental set-up}

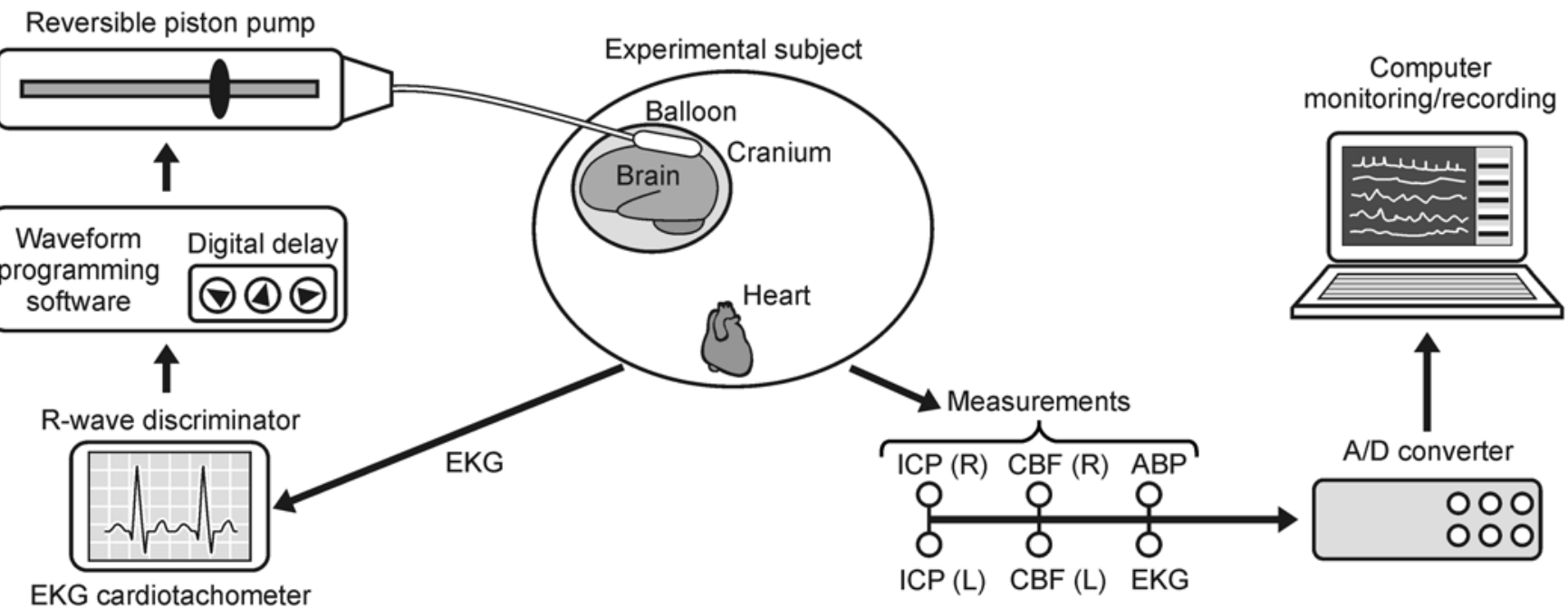

FIG. 1. Schematic of the Cadence system and experimental setup showing the major components used to operate the system (pump, EKG cardiotachometer, digital delay) and the output data collection parameters (ICP, CBF, ABP, and EKG). $L=l$ eft; $R=$ right.

a silicone catheter, which was externalized via tunneling and attached to the pump system and a pressure-monitoring device.

To effect appropriate intracranial volume changes, balloons of various sizes (0.1-1.0 ml) were produced (Fig. 2). The prototype balloons were manufactured using a dipcoating method with an alloy mandrel, in a silicone rubber material, specifically chosen to be biocompatible and flexible, but inelastic. The catheters connecting the pump to the balloon were also inelastic to prevent the attenuation of the waveform prescribed by the pump.

\section{Programmable Pump}

Balloon volume is controlled by the oscillating pump in a closed-air system to allow rapid bidirectional volume transfer. The prototype device was assembled at the Mechanical Prototype Core and Electronics Core at Medical Device Solutions, Cleveland Clinic. The motion of

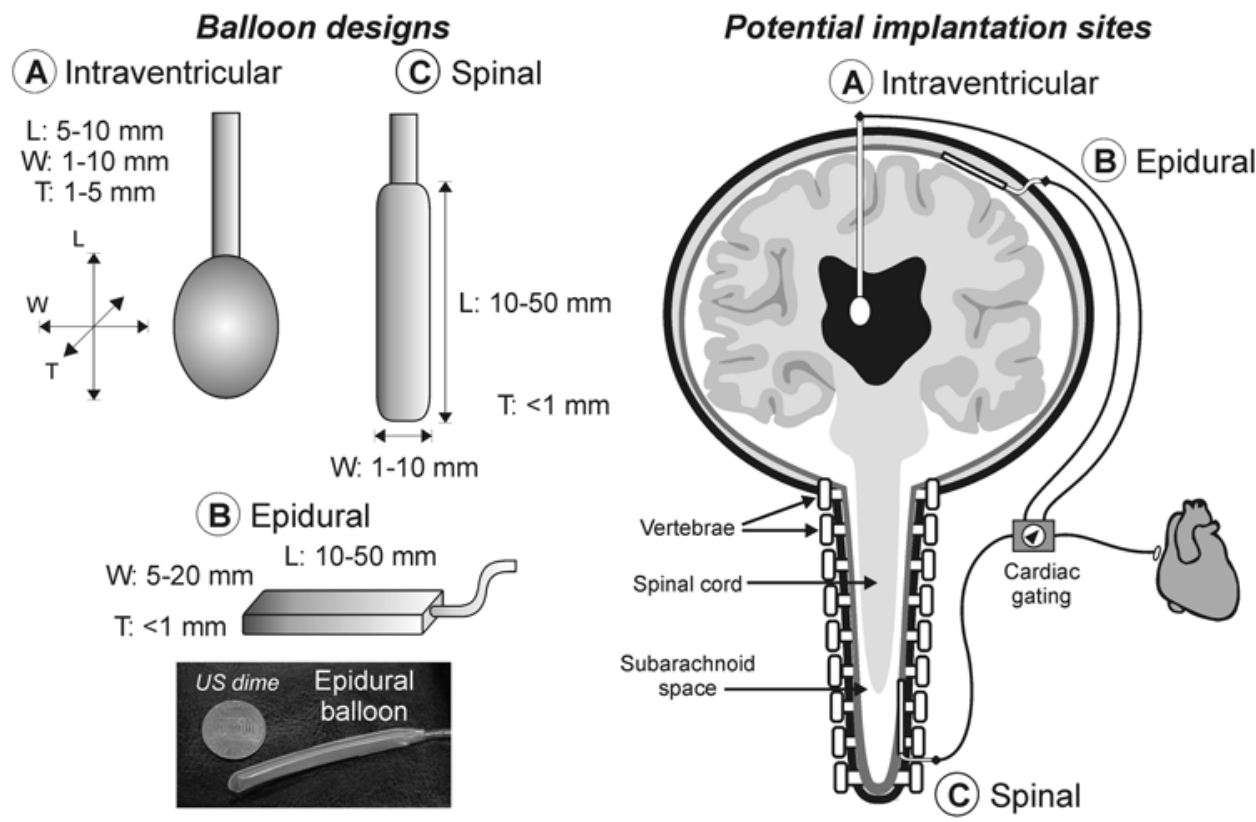

FIG. 2. Illustration showing different balloon designs and implantation sites. Different balloon designs (left) include intraventricular $(A)$, epidural $(B)$, and spinal $(C)$ embodiments; different implantation sites (right) can include intraventricular $(A)$, epidural $(B)$, and spinal $(C)$. In the present experiments, a cranial epidural balloon was used; the photograph (bottom left) shows an example (with a US dime for size reference). $L=$ length; $T$ = thickness; $W=$ width. 
the pump piston was controlled by dedicated software running on a personal computer. Based on input from a 3-lead electrocardiogram (EKG) signal and a commercial R-wave detector (CWE Inc.), the software synchronizes the forward and backward motion of the piston to the cardiac cycle on a beat-to-beat basis. The pump has a 24$\mathrm{mm}$ diameter, 50-mm stroke length air-filled antifriction cylinder (Airpot Corporation), with a piston driven by a stepper motor using a ball screw linear actuator (Model EZC6-05, Oriental Motors Co., Ltd), via a ball-joint interconnection. The stepper motor is, in turn, controlled and driven by a dedicated motor controller/driver (Oriental Motors Co., Ltd).

\section{Cardiac Gating and Balloon Waveform Programming}

The programmable system uses R-wave detection as the trigger for initiation of a balloon inflation/deflation cycle. The time delay from the R-wave, and speed and duration of inflation can be adjusted. The delay from the $\mathrm{R}$-wave to initiation of inflation/deflation can be specified at the millisecond level, and the waveform is adjustable by specifying a variable number of consecutive steps of piston movement. Each step is defined by the incremental displacement of the piston (percentage of total volume) and the duration (percentage of the cardiac cycle) in which to carry out the motion. Thus, the theoretical amplitude of the balloon volume variation $(\Delta \mathrm{BV})$ of the waveform is the direct result of the maximum volume displacement achieved over the cardiac cycle. By actively modifying the amplitude and direction of volume variation, as well as the velocity, timing, and duration of inflation within the cardiac cycle, the system can be used to alter the ICP waveform. The pressure in the air-filled catheter between the pump cylinder and the balloon was recorded as an indicator of balloon volume variation caused by the pump, for monitoring changes and potential leakage during the experimental procedures and for offline analysis.

\section{Implantation and Experimental Setup}

These pilot experimental studies used 19 male, random-source hounds (Canis familiaris) aged approximately 8-9 months (i.e., young adults) weighing approximately $25-30 \mathrm{~kg}$. The balloons were acutely implanted for $8-10$ hours, although the longest persistent balloon activity was 4 hours. The canine model was used because of its relative size and anatomical similarities of brain, cerebrovascular, and CSF systems to humans. All canines were obtained from licensed suppliers (Marshall Farms and Hodgins Kennel), quarantined for a minimum of 7 days before study entry, and maintained in the Cleveland Clinic's fully accredited animal care facility under the guidelines of the Guide for the Care and Use of Laboratory Animals. While the exact experiments performed varied between canines, the overall implantation and experimental setup was the same, and several of the canines were used in more than 1 of the analyses presented in this paper.

Anesthesia was induced using sodium pentothal (20 $\mathrm{mg} / \mathrm{kg}$, intravenously) and the animals were then intubated and placed on a ventilator with isoflurane gas $(1.0 \%-1.5 \%)$ used to maintain anesthesia throughout the study. Presur- gically, animals were given phenytoin $(5 \mathrm{mg} / \mathrm{kg}$, intravenously) to prevent postoperative seizures, dexamethasone $(0.25-1.0 \mathrm{mg} / \mathrm{kg}$, intravenously) to reduce inflammation, glycopyrrolate $(0.01 \mathrm{mg} / \mathrm{kg}$, intravenously) to reduce respiratory secretions, and gentamicin ( $3 \mathrm{mg} / \mathrm{kg}$, intravenously) and cefazolin ( $1 \mathrm{~g}$, subcutaneously) to prevent infection.

Animals were placed in a stereotactic head frame in prone position. A single sagittal incision was made at the midline, and the skin, fascia, and temporalis muscle were retracted to expose the skull. Balloon catheters and intracranial ICP and CBF sensors were implanted under sterile conditions. The balloons were inserted into the cranial epidural space above the Sylvian sulcus, along a posterior-toanterior axis, through an $8-\mathrm{mm}$ bur hole made by a twist drill. Additional bur holes and dural openings were made to insert ICP and CBF monitoring probes bilaterally into the parenchyma (at a depth of about 1-2 cm) and made watertight by filling the epidural space with saline to remove air, and repairing the skull using acrylic paste to prevent direct communication between the atmosphere and intracranial space. Probes were kept in position throughout the duration of intraoperative testing of the Cadence system. ICP sensors used were commercially available (Camino 110-4BT, Integra Lifesciences Inc., or Codman Microsensor, Johnson \& Johnson Inc.).

CBF was measured bilaterally in the dorsolateral prefrontal cortex using either surface or needle laser Doppler probes (BLF21D Dual Channel Flowmeter, NSABLPHN24 BLF Laser Doppler Flowprobe, Transonic Systems Inc.) and/or thermal diffusion probes (Hemedex Cerebral Blood Flow Monitoring System, Bowman Perfusion Monitor, QFlow 500 probe; Hemedex). CBF was measured continuously in approximately $1-\mathrm{mm}^{3}$ volume of brain parenchyma for laser Doppler $\mathrm{CBF}\left(\mathrm{CBF}_{\mathrm{LD}}\right.$; tissue perfusion units [TPUs]) and perfusion in a volume $<0.3 \mathrm{~mm}^{3}$ at a frequency of $1 \mathrm{~Hz}$ for thermal diffusion $\mathrm{CBF}\left(\mathrm{CBF}_{\mathrm{TD}}\right.$; absolute units of $\mathrm{ml} / \mathrm{min} / 100 \mathrm{~g}$ tissue). In the proof of concept study, $\mathrm{CBF}$ velocity $\left(\mathrm{CBF}_{\mathrm{v}}\right)$ was monitored using transcranial Doppler ultrasound (Viasys WinTC, Viasys).

In addition to ICP and blood flow, the EKG, arterial blood pressure $(\mathrm{ABP})$, breathing, temperature, arterial blood gases, and $\mathrm{pH}$ were monitored. The blood gases and $\mathrm{pH}$ were maintained within normal ranges $(\mathrm{pH} 7.4$, partial pressure of carbon dioxide $35-45 \mathrm{~mm} \mathrm{Hg}$, partial pressure of oxygen $95-100 \mathrm{~mm} \mathrm{Hg}$ ) mechanically by controlling the ventilation volume and frequency, and body temperature was maintained with a thermal blanket $\left(37^{\circ} \mathrm{C} \pm 3^{\circ} \mathrm{C}\right)$ and intravenous infusion of a saline solution of Ringer's lactate at a rate of $25 \mathrm{ml} /$ hour (throughout surgery).

\section{Data Processing}

All manipulations were monitored in real time with a multichannel analog-to-digital translating (A/D) data acquisition system (PowerLab version 5.3.2, ADInstruments Pty Ltd), and visual feedback could be used to perform online adjustment of system settings. ICP, CBF, EKG, ABP, and Cadence balloon pressure were recorded in real time, at sampling rate of $200 \mathrm{~Hz}$. For each activation session, a baseline segment recorded immediately prior to system activation was compared with the same duration of activa- 
tion. All data segments were analyzed using a specifically designed automated MATLAB algorithm (R2006b, The Mathworks, Inc.). This section summarizes the data analysis; further details are presented in the Appendix.

For waveform analysis, ICP and laser Doppler blood flow signals were high-pass filtered to isolate the cardiacrelated variations from respiratory and other slow waves. The filtered signals were only applied to the detection of peaks and troughs related to the cardiac cycles, and amplitude calculations; single ICP or CBF values, as well as mean values, were determined from unfiltered data.

A threshold-based peak detection algorithm for the R-wave of the EKG determined the start of each cardiac cycle. Systolic ICP was determined as the maximum ICP, i.e., the peak ICP of the cardiac cycle; however, as deflation protocols often caused a dip in the ICP during systole, systolic ICP for these protocols was defined as the lowest local extreme during the relevant part of the cardiac cycle (i.e., either a peak or a trough). The detection of the systolic ICP was limited to the first half of the cardiac cycle starting after the diastolic ICP of the baseline measurement; additionally, it was restricted to being at least 20 milliseconds after the initiation of the balloon volume change for the activation segments (Fig. 3). The systolic ICP pulse was defined as the systolic ICP minus the diastolic ICP.

To illustrate the different balloon waveforms and the ICP waveforms resulting from Cadence activation, ensemble averaging (cycle to cycle) of the different signals was applied to each baseline and activation segment. These ensemble-averaged waveforms were only used for illustration; all determinations of ICP and CBF parameters were performed on nonaveraged data.

Long-term (4-hour) activation data were analyzed and averaged for 4-minute blocks immediately prior to activation, throughout continuous activity, and immediately

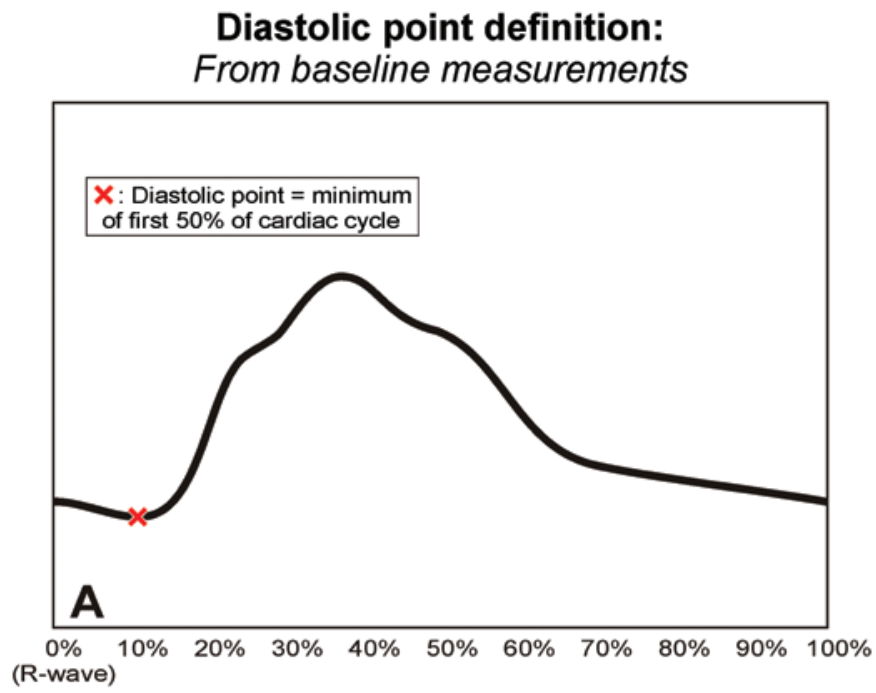

Systolic ICP \& ICP amplitude definition: Baseline \& inflation protocols

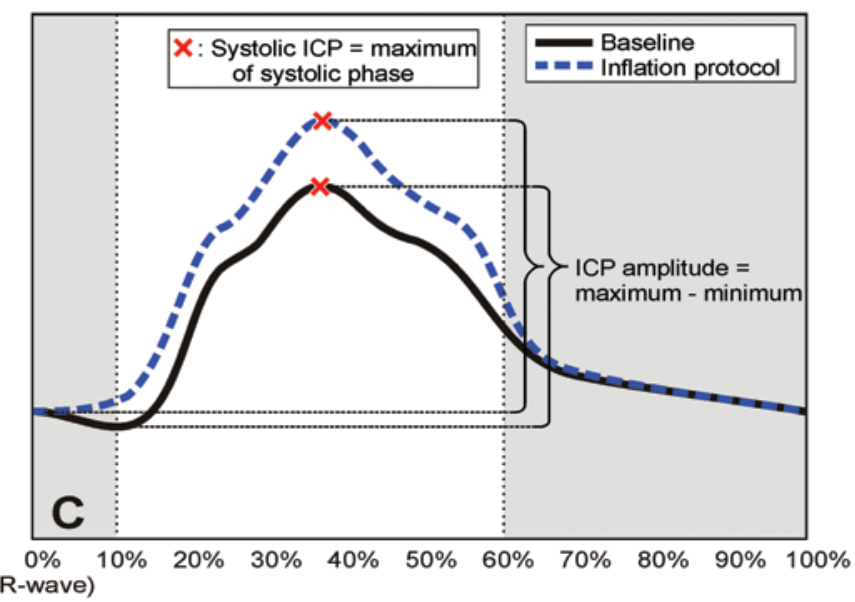

\section{Systolic phase \& diastolic ICP definition: Baseline, inflation \& deflation protocols}

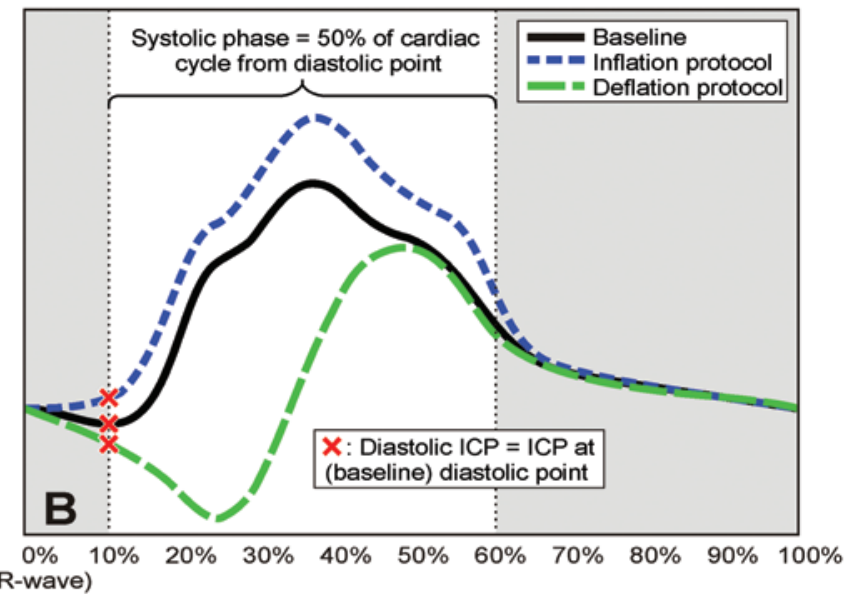

Systolic ICP \& ICP amplitude definition: Deflation protocols only

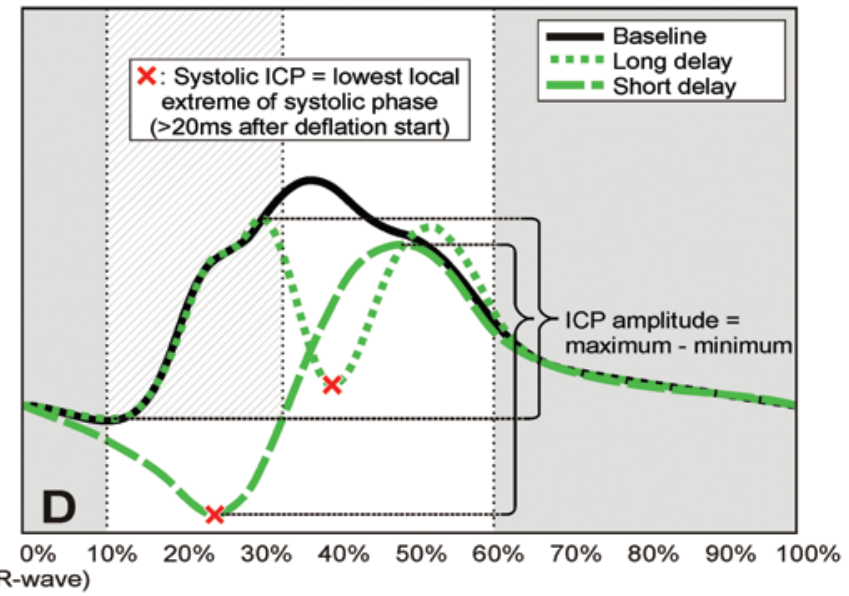

FIG. 3. Illustration of ICP parameter calculations. The diastolic point was always determined using the baseline measurements (A), and this defined the diastolic ICP and the region in which to determine the systolic ICP for all protocols (B). The systolic ICP definition differed for inflation (blue) and deflation (green) protocols ( $C$ and D). Panel D shows two different versions of deflation protocols, one with short balloon cycle delay and one with longer delay. 
following deactivation for the synergistic inversion protocol. Comparisons were made between 4-minute blocks immediately before and after activation and deactivation, and the 4-minute blocks of the continuous activity were analyzed for trends. Blocks in which the balloon cycle delay, balloon pressure amplitude, or heart rate differed more than $10 \%$ from the median for more than $25 \%$ of the cardiac cycles were excluded.

\section{Results}

\section{Effectiveness of ICP Manipulation}

To demonstrate the efficacy of ICP waveform modulation, the first proof of concept study involved 3 canines in which the Cadence system was used with the balloons inserted into the cranial epidural space. With varying degrees of volume variation and R-wave delay, the results ranged from no ICP pulse wave modification to more than 2 -fold alteration of the pulse amplitude. The initial systemoff balloon state (inflated or deflated) also changed the waveform effect. For example, with the balloon starting in the inflated state the ICP lowering effect of the deflation at systole predominated (Fig. 4). Importantly, the effect on waveform did not significantly change the heart rate, or mean arterial or ICP pressures. The slower ICP variation due to respiration was retained and the diminished ICP waveform with Cadence activation was reversible when Cadence was turned off (Fig. 4).

\section{Characterization of Direct ICP Manipulation}

Based on a range of tests conducted during the serial development of the method (in a total of 16 canines), we were able to determine the effects of specific balloon cycle parameters (i.e., balloon volume, activation delay, inflation/deflation) and thus design balloon protocols for direct manipulation of the ICP waveform within the cranium. Due to the variation in the balloon protocols and probe placements during the development, statistical group analysis of the data was not appropriate.

Observed ICP effects of Cadence system activation were consistently bilateral, although the response strength varied with respect to balloon location (local vs distal); effects observed in the contralateral hemisphere were approximately $70 \%-90 \%$ of the ipsilateral/local effect (5 canines).

\section{Effect of Synergistic Versus Antagonistic Protocols}

In the inflation protocols the balloon started in an inflated state and was deflated during systole, theoretically increasing intracranial volume capacity precisely as arterial blood is entering the cranium. Therefore, these protocols were synergistic to the natural physiological activity of the cardiovascular system. Protocols in which the balloon was initially in a deflated state and every cycle started with inflation during systole, should theoretically increase the resistance to the arterial volume increase, and were thus considered antagonistic to the cardiovascular activity.

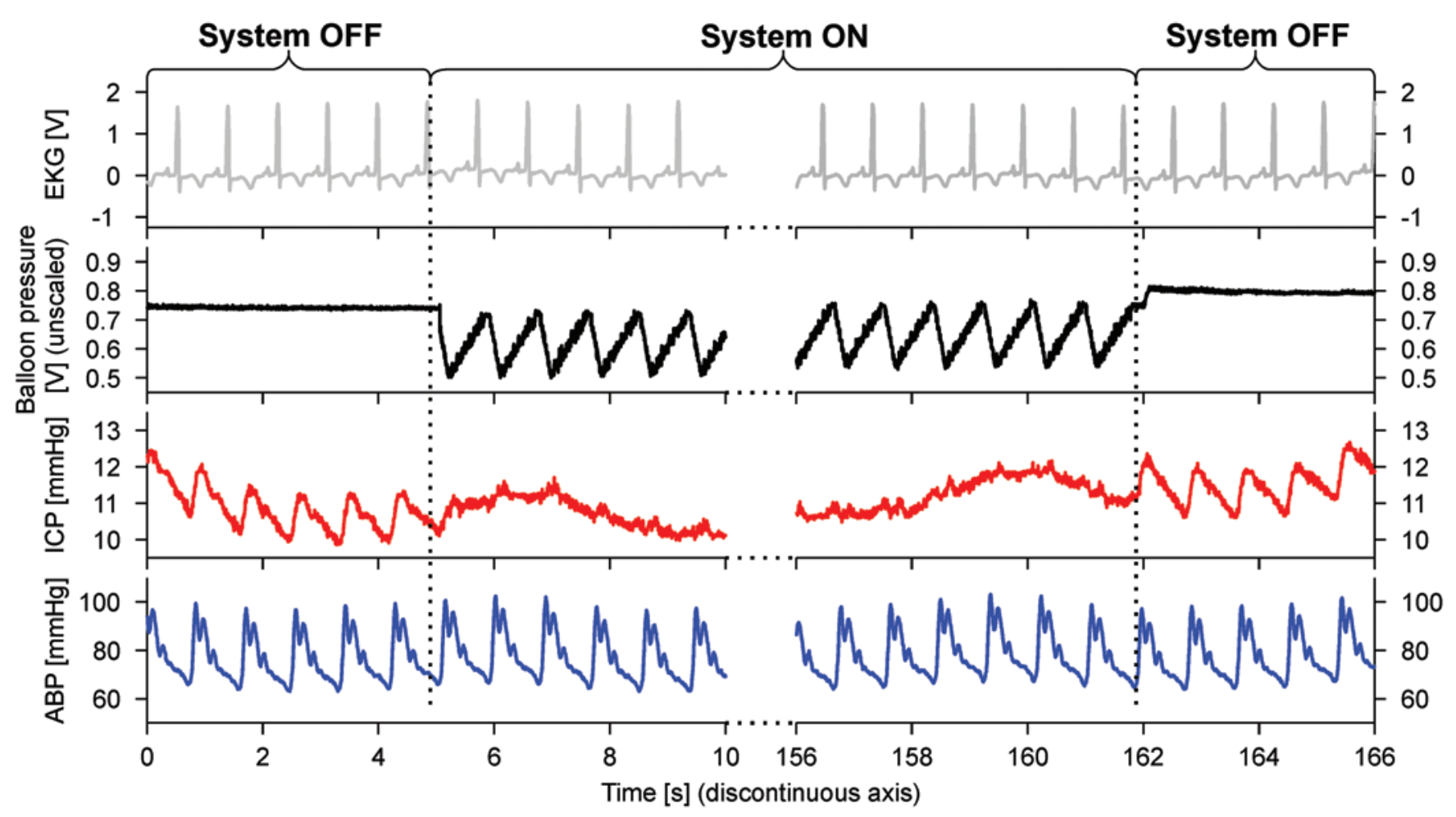

FIG. 4. Effects of cranial epidural Cadence balloon activation. The programmed balloon volume change was $0.8 \mathrm{ml}$ (deflation) with activation $170 \mathrm{msec}$ after the R-wave. The ICP (red) pulse amplitude was substantially reduced (approximately $70 \%$ ) immediately after Cadence balloon activation (black). The effect was reversed at deactivation, about 2.5 minutes later. Note that EKG and ABP did not show any acute changes during Cadence activation. 
Typical effects of synergistic and antagonistic protocols are shown in Fig. 5A and B and Table 1.

\section{Effect of Timing Delay Between R-Wave and Balloon Activation}

Using synergistic balloon protocols, variable delays (approximately 40-200 msec) of EKG R-wave-triggered inflation/deflation (i.e., activation delay) were investigated in 7 canines. Overall, systolic ICP was reduced at all delays but, on average, the effect was reduced by $1.5 \mathrm{~mm} \mathrm{Hg}$ per 100-msec increase in the delay (synergistic protocols).

\section{Effects of Balloon Volume Change on ICP Waveform}

Increases in the balloon volume changes $(\Delta \mathrm{BV}$, theoretically approximately $0.1-1 \mathrm{ml}$, synergistic cycles), while maintaining all other variables (e.g., activation delay) constant for each canine $(n=6)$, caused the effect on systolic ICP to increase linearly (a mean $1.4 \pm 0.9 \mathrm{~mm} \mathrm{Hg}$ per 10 $\mathrm{mm} \mathrm{Hg}$ increase in balloon pressure amplitude).

For synergistic protocols with small $\Delta \mathrm{BV}$, the systolic ICP decreased by small enough magnitudes that the ICP pulse amplitude was reduced (range -0.5 to $-2 \mathrm{~mm} \mathrm{Hg}$ ).
A

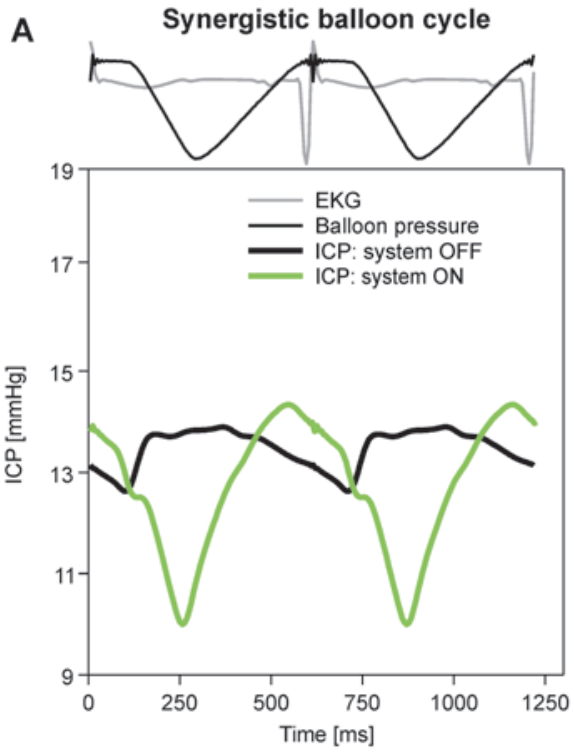

C
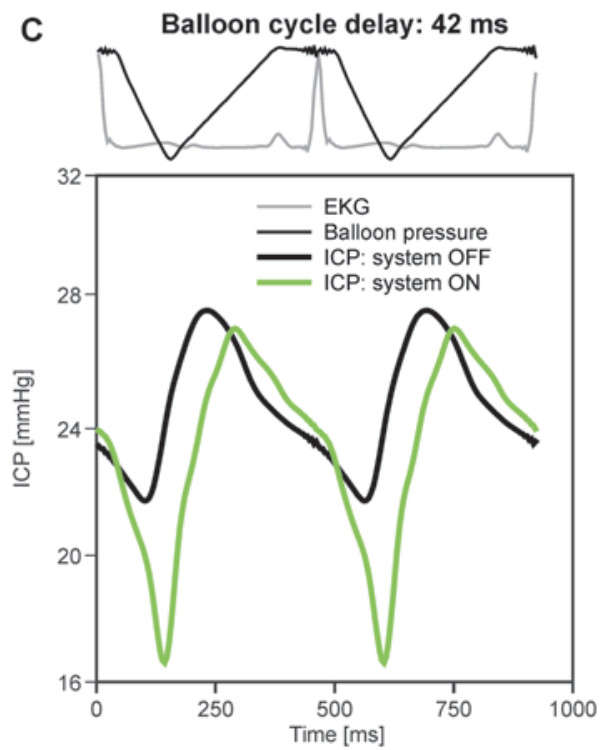

B
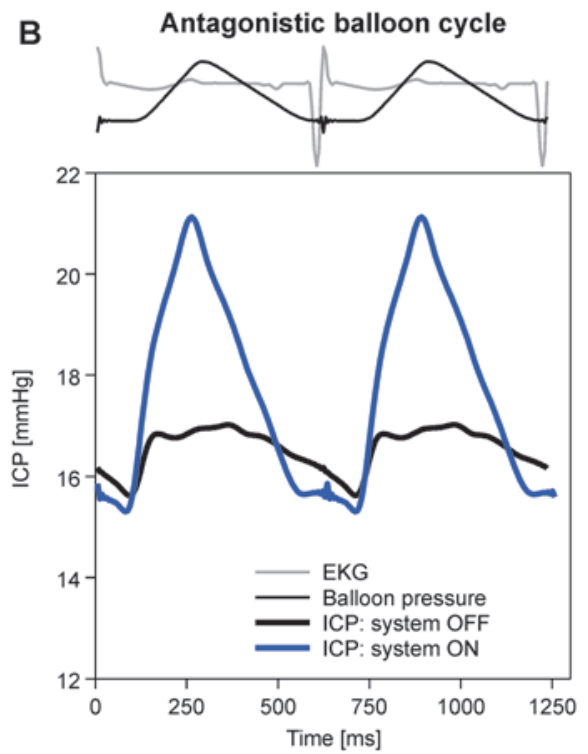

D

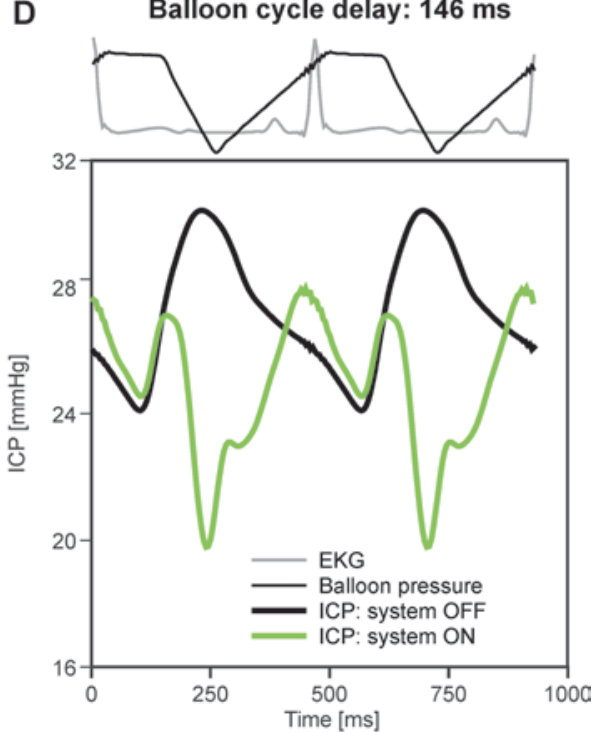

FIG. 5. Examples of ICP waveforms produced by synergistic and antagonistic balloon cycles, and different activation delays (ensemble-averaged data). With synergistic activation (A), i.e., detection of EKG (gray) R-wave triggers balloon deflation (balloon pressure; thin black line), the ICP waveform (green) was altered from the baseline (black line) with a downward deflection in systole and an increase above baseline in diastole. As a result, ICP pulse pressure was increased compared with baseline. With antagonistic activation (B), increase in balloon pressure at systole led to augmentation of the ICP pulse amplitude (blue line). Total balloon volume variation was the same for the two protocols (theoretically $1 \mathrm{ml}$ ). Note that both the baseline and the resultant ICP waveform have a higher mean for the antagonistic balloon cycle, likely due to normal physiological variation in ICP, as the protocols were not run one after the other. Activation with short (C; $42 \mathrm{msec}$ ) and long (D; $146 \mathrm{msec}$ ) R-wave balloon-activation delays resulted in different effects depending on the specific timing of the balloon deflation with regard to the baseline ICP waveform affecting the resultant ICP waveform. 
TABLE 1. Effects on mean ICP and ICP waveform with synergistic (deflation in systole) or antagonist (inflation in systole) protocols*

\begin{tabular}{lccc}
\hline & & \multicolumn{2}{c}{ Change w/ Activation } \\
\cline { 3 - 4 } Variable $(\mathrm{mm} \mathrm{Hg})$ & Baseline (cadence off) & Inflation w/ Systole & Deflation w/ Systole \\
\hline Mean ICP & $12-17$ & -3 to $2(-18 \%$ to $13 \%)$ & 0 to $-3(0$ to $-18 \%)$ \\
\hline Systolic ICP pulse & $1.1-1.4$ & $4-16(360 \%-1200 \%)$ & -0.5 to $-19(-5 \%$ to $-1500 \%)$ \\
\hline ICP pulse amplitude & $1.2-1.6$ & $4-19(300 \%-1400 \%)$ & -2 to $21(-25 \%$ to $1600 \%)$ \\
\hline
\end{tabular}

* R-wave activation delay $=40-200 \mathrm{msec}$. Cardiac-gated balloon volume change $0.1-1 \mathrm{ml}$. ICP effects measured ipsilateral to the balloon.

Percentages are relative to baseline.

With larger $\triangle \mathrm{BV}$, the systolic ICP decreased below the diastolic value, thus inverting the systolic ICP pulse, and resulting in increased ICP pulse amplitude (range 0.5-21 $\mathrm{mm} \mathrm{Hg}$ ) due to the systolic ICP change and an additional peak at balloon re-inflation (Fig. 5).

\section{Balloon Protocol Design for Direct ICP Waveform Manipulation}

From the range of experiments presented here, 3 sets of balloon waveform settings were defined, corresponding to different effects on the ICP waveform. Balloon cycle protocols could thus be designed for direct manipulation of the ICP waveform in the form of ICP reduction, ICP inversion, and ICP augmentation (Fig. 6).

\section{CBF and CBF Velocity Changes in Response to ICP Waveform Manipulation}

To assess the direct effect of ICP manipulation on CBF and $\mathrm{CBF}$ velocity, the Cadence system was activated with balloons in the cranial epidural space in conjunction with thermal diffusion $\left(\mathrm{CBF}_{\mathrm{TD}}\right)$ and laser Doppler $\left(\mathrm{CBF}_{\mathrm{LD}}\right)$ monitoring of $\mathrm{CBF}$, and transcranial Doppler $\left(\mathrm{CBF}_{\mathrm{v}}\right)$ monitoring of CBF velocity. Overall, CBF was shown to increase more with activation of inversion than augmentation protocols (Fig. 7 upper). While there was a bilateral increase in
CBF with Cadence system activation, a greater effect was observed in the ipsilateral hemisphere than distally (contralateral $<50 \%$ of ipsilateral increase). $\mathrm{CBF}$ increases were observed consistently with ICP inversion protocols, while ICP reduction and ICP augmentation resulted in milder, less consistent increases in CBF (Fig. 7 lower).

Similarly, in the proof of concept study, a modest increase in $\mathrm{CBF}_{\mathrm{V}}$ was observed with Cadence system activation using a deflation protocol in the right $(+18 \%)$ and left $(+9 \%)$ siphons, and a dramatic increase in the basilar artery $(+39 \%)$ under normal physiological conditions (Fig. 7 upper; $\mathrm{n}=1)$.

\section{Long Term (4-Hour) Effectiveness of Cadence System Activity on ICP and CBF}

Four-Hour Cadence Activation

While initial studies were aimed at acute or short-term effects (i.e., minutes) of Cadence activation, long-term (4hour) activation was further investigated in 10 animals (ICP inversion). The animals remained physiologically stable during Cadence activity, for both short and longer (up to 4-hour) periods, with mean systemic blood pressure remaining within $\pm 10 \mathrm{~mm} \mathrm{Hg}(13 \%)$ of the baseline value (before activation) and the heart rate within $\pm 17 \mathrm{bpm}$ $(16 \%)$.

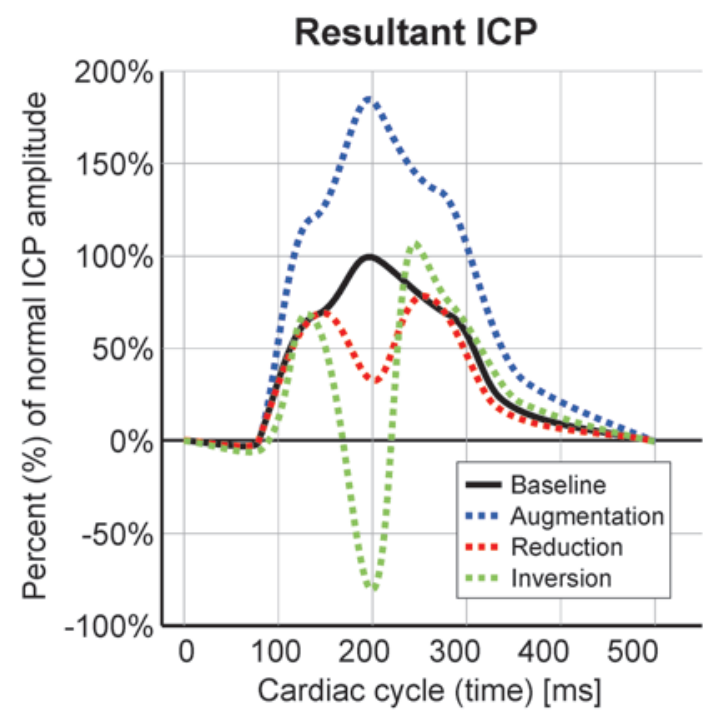

FIG. 6. Cadence balloon cycles and ICP waveforms of the 3 ICP manipulation protocols. Using synergistic cycles where the balloon is deflated during systole (left), to produce a smaller (red line) or larger (green line) volume change $(\Delta \mathrm{BV})$, results in reduction or inversion of the ICP waveform (right). The antagonistic protocol (blue line), i.e., balloon inflation in systole, results in augmentation of the ICP pulse wave. 

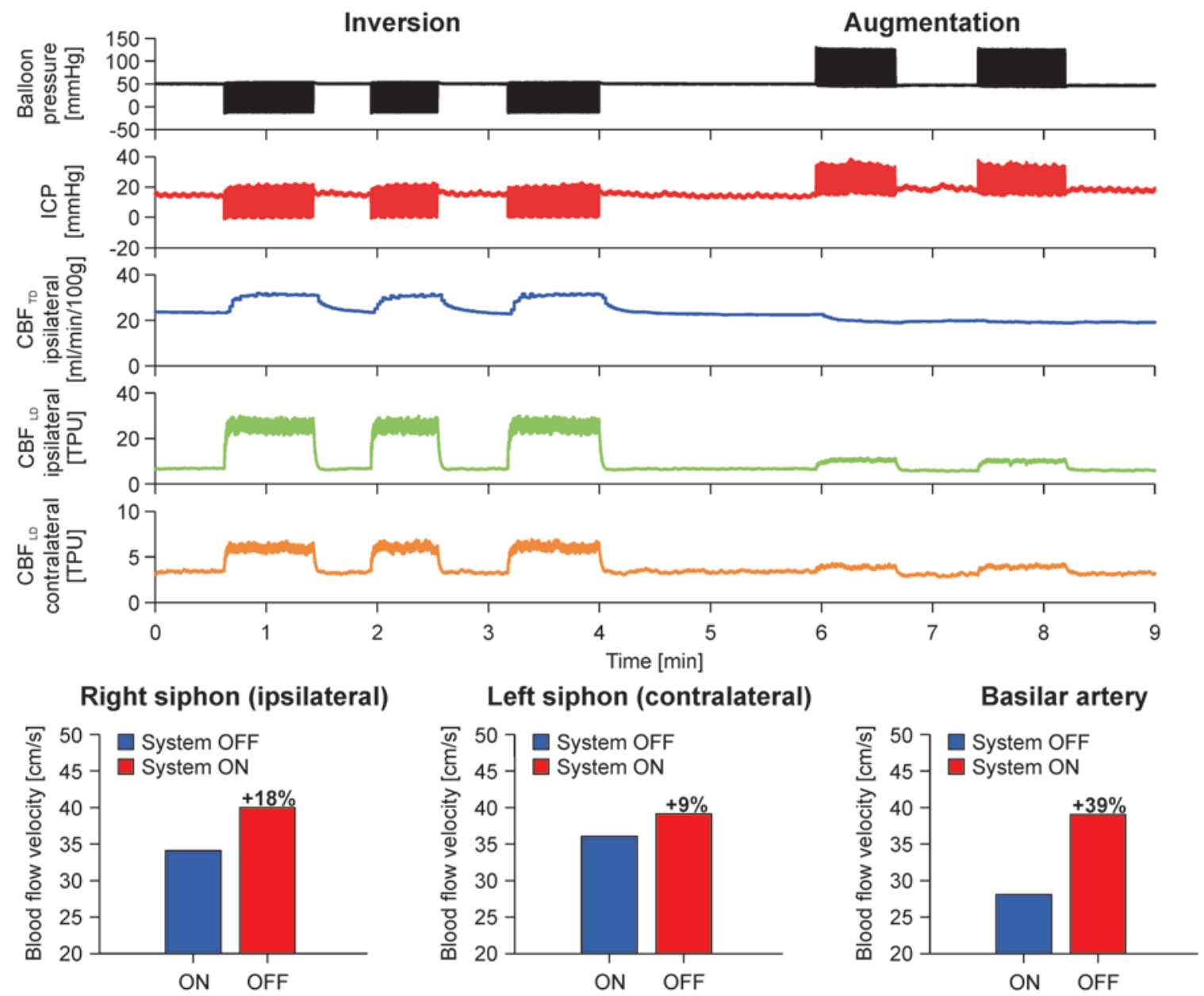

FIG. 7. CBF and CBF velocity changes with direct ICP manipulation. Clear increases were observed in $\mathrm{CBF}_{\mathrm{LD}}$ (green and orange lines), as well as in $\mathrm{CBF}_{\mathrm{TD}}$ (blue line) in response to inversion of ICP (red line), i.e., deflation of the balloon at systole (balloon pressure; upper left, black line). For augmentation of ICP, $\mathrm{CBF}_{\mathrm{LD}}$ increases were smaller, and in this example no $\mathrm{CBF}_{\mathrm{TD}}$ increase was observed (upper, right side). Cadence activation with a deflation protocol in 1 animal from the proof of concept study (lower) resulted in increases in mean $\mathrm{CBF}_{\mathrm{v}}$ in the carotid siphons (left and right) and basilar artery (percentages indicate increases relative to baseline).

The effects of Cadence activation were persistent over 4 hours (Fig. 8), with no consistent trends of degradation identified in the systolic ICP, ICP pulse amplitude, mean $\mathrm{ICP}$, or mean $\mathrm{CBF}_{\mathrm{LD}}$; while the average increase in mean $\mathrm{CBF}_{\mathrm{TD}}$ decreased over the 4 hours, this effect was not statistically significant (the average of the 4-hour slope was not significantly different from zero). The observed immediate changes in the ICP and CBF upon activation (Table 2) were reversed when the system was deactivated (no significant difference in the magnitude of the effects).

\section{Gross Anatomy and Histology}

Gross inspection revealed no signs of cortical contusion or hemorrhages in or around the brain and CSF spaces after 4-hour Cadence activity. A minimal amount of epidural bleeding corresponding to the area above balloon catheter placement was observed, likely related to the insertion. While no ventriculomegaly or atrophy was observed in any animal, a small amount ( $<1-2 \mathrm{~mm}$ ) of cortical compression or distortion was observed in a few animals (Fig. 9A and B). Preliminary results from standard staining methods (histological: cresyl violet and $\mathrm{H}$ \& E; immunocytological: glial fibrillary acidic protein, neuron-specific enolase, S100B) showed no signs of cell death, apoptosis, glial scarring, infection, or inflammation in the cortex adjacent to balloon location (Fig. 9C and D). In addition, no signs of cellular injury in distal regions of the cortex or hippocampus were found when comparing animals that underwent different ICP waveform manipulations (i.e., inversion, augmentation, and reduction protocols).

\section{Discussion}

The method and device presented in this paper were shown to effectively produce different changes in the ICP pulse wave, varying with the design of the balloon inflation pattern, when the balloon was placed in the intracranial epidural space. Importantly, the use of the Cadence device was well tolerated, and showed an encouraging lack of effect on the mean ICP level and on the peripheral physiology. The ICP pulse wave could be reduced in amplitude when using the system synergistically to the cardiac activ- 
TABLE 2. Effects on ICP, ABP, and heart rate with Cadence system activation (synergistic protocols) and deactivation after 4 hours*

\begin{tabular}{cccc}
\hline Variable & Baseline (Cadence off) & Change w/ Activation & Change w/ Deactivation \\
\hline ICP variables $(\mathrm{mm} \mathrm{Hg})$ & & & \\
\hline Mean ICP & $9.1-23.0$ & -3.1 to $1.0(-14 \%$ to $9 \%)$ & -0.3 to $7.3(-3 \%$ to $30 \%)$ \\
\hline Systolic ICP pulse & $0.6-8.4$ & -0.6 to $16.3(-95$ to $-536 \%)$ & $1.2-21.0(114 \%-447 \%)$ \\
\hline ICP pulse amplitude & $0.6-8.4$ & $0.2-10.9(25 \%-477 \%)$ & -0.3 to $-14.7(-12 \%$ to $-398 \%)$ \\
\hline Systemic variables & & & -6.4 to $3.3(-11 \%$ to $4 \%)$ \\
\hline Mean ABP $(\mathrm{mm} \mathrm{Hg})$ & $51.2-78.8$ & -0.2 to $3.9(0-7 \%)$ & -11 to $1(-10 \%$ to $1 \%)$ \\
\hline Heart rate $(\mathrm{bpm})$ & $78-133$ & -2 to $6(-2 \%$ to $8 \%)$ &
\end{tabular}

* ICP effects measured ipsilateral to the balloon. Percentages are relative to baseline.

ity, i.e., deflating the balloon at systole, and increasing this deflation caused inversion of the waveform by decreasing the systolic ICP below the diastolic level. Using the system antagonistically to the cardiac activity produced an increase of the ICP pulse wave by raising the systolic ICP. Furthermore, it was shown that this method had potential for increasing the CBF with any of the 3 above-mentioned types of ICP pulse wave modifications, although inversion of the ICP pulse wave produced the most consistent $\mathrm{CBF}$ increase.

The ICP and CBF effects were observed in both cerebral hemispheres, suggesting that the Cadence system may be used to modify the ICP and other physiological processes in the entire intracranial space, although the response may be strongest proximal to the implanted balloon.

The results suggested that using the system had almost instantaneous results on the ICP, with modifications of the ICP pulse wave occurring during the same cardiac cycle as the balloon inflation/deflation, and disappearing or changing with the next cardiac cycle if the system was deactivated or the balloon cycle altered. These immediate responses to changes in the balloon volume variation are consistent with the scientific understanding of the pressure and volume relationships within the intracranial space, as described by the Monro-Kellie doctrine and the Windkessel mechanism., ${ }^{7,810,13,19,25,27}$ While ICP effects were observed immediately, the $\mathrm{CBF}$ changes developed over several cardiac cycles (Fig. 7 upper). Based on the laser Doppler measurements, however, some part of the CBF increase, or decrease after deactivation, did occur within the first cardiac cycle. Both ICP and CBF effects persisted with Cadence activation for at least 4 hours, and the effects were still reversible, similar to the reversibility noted with shorter activation periods, suggesting that no lingering changes in ICP or CBF were produced even when the activation persisted for hours.

Based on these initial results, the Cadence system may have potential for use in the study of the effects of ICP pulse wave changes on cranial physiological processes such as brain morphology, ventricular size, circulation and clearance/absorption of CSF, and drug delivery.

The feasibility of using cardiac-gated intracranial implants for therapeutic ends is unknown; however, given the suggested roles of the pulsatile dynamics of blood and CSF,
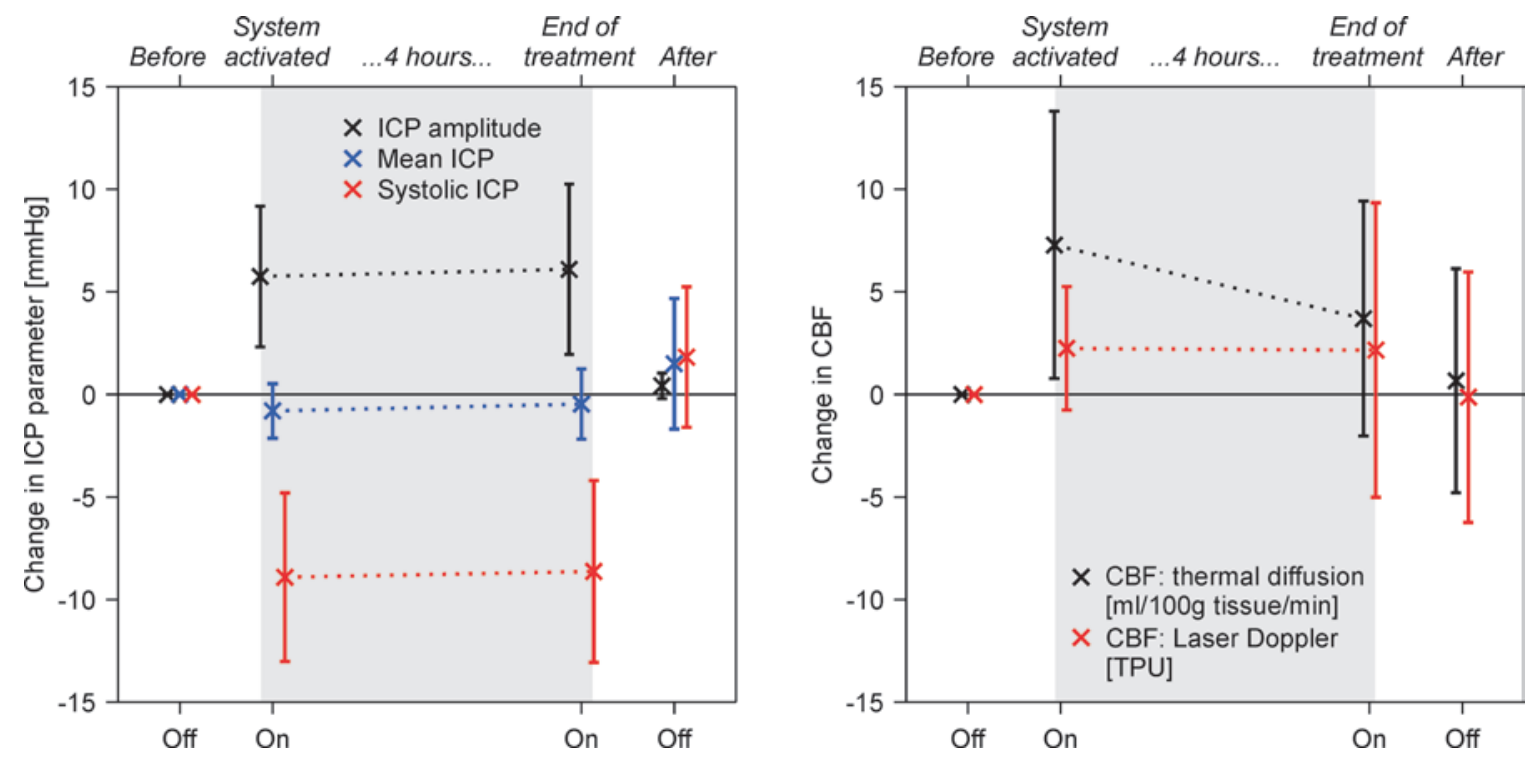

FIG. 8. ICP and CBF effects of 4 hours of Cadence activation ( $n=10,4$-min averages). Left: The effects on ICP amplitude (black), mean ICP (blue), and systolic ICP (red) were stable over the 4 hours. Right: Similarly, CBF was stable, although a nonsignificant reduction of the average $\mathrm{CBF}_{\mathrm{TD}}$ increase was observed. After deactivation, all parameters returned to levels close to the baseline values, although some variability can be observed for the CBF values. 

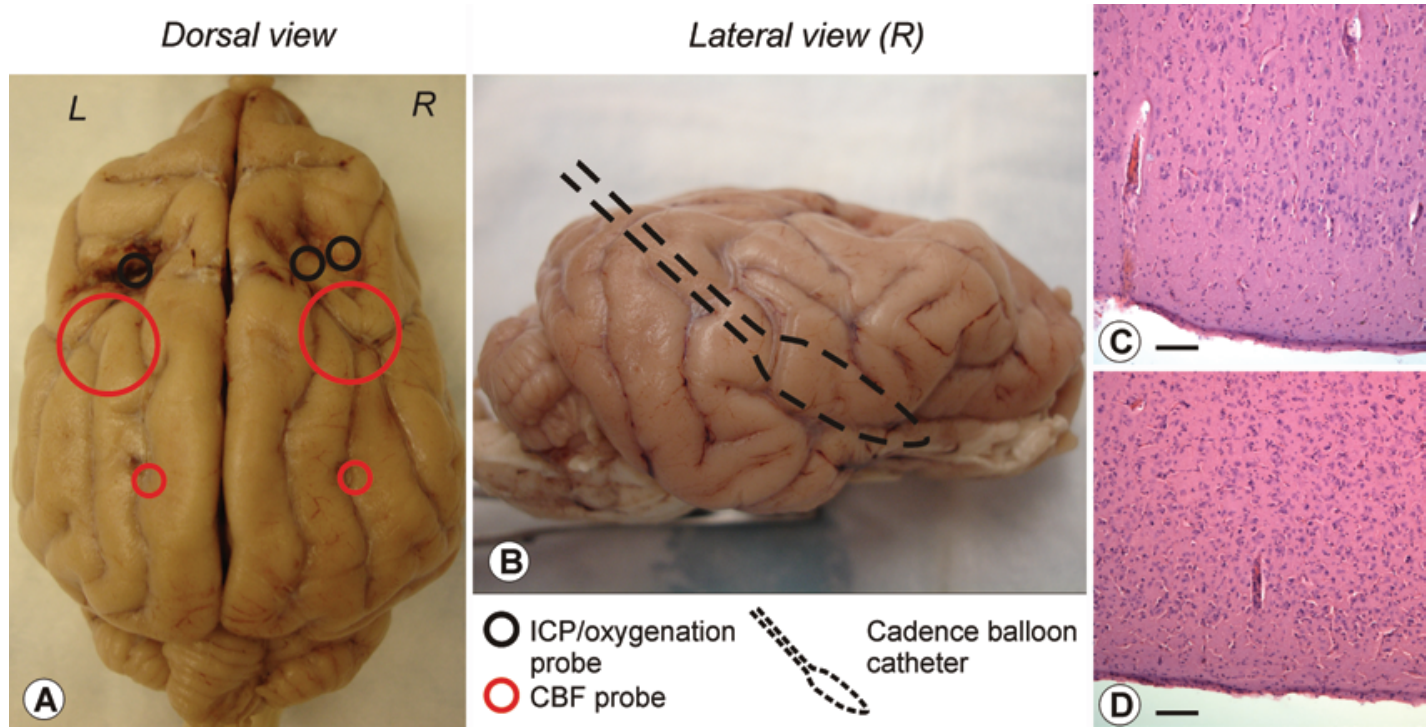

FIG. 9. Photographs showing gross brain pathology and histology. Dorsal (A) and right lateral (B) views of the brain and photomicrographs $(H \& E)$ of the right parietal cortex, distal to the balloon $(C)$ and underneath the balloon (D), show little to no injury after 4-hour activation. Bar $=100 \mu \mathrm{m}$.

these ends may include the restoration of CBF in acute or chronic low blood flow states, specifically in stroke or trauma, given there is still enough fluid space to modulate the intracranial volume. Because ventriculomegaly may be generated by a pulsatile process, ${ }^{2,9}$ modulation of ventricular and cyst size may also be considered. Finally, improved CSF solute distribution and clearance brought about by CSF pulsatility changes may be useful in the treatment of neurodegenerative diseases and tumors..$^{11,14}$

However, even if positive effects are observed, the optimal balloon placement and shape, inflation pattern, and pump system have yet to be determined. The significant results reported here with epidural placement raise the question of more efficacious CSF effects directly in CSF spaces such as the ventricles and cisterns. Furthermore, although these initial studies showed that a brief application did not show significant deleterious physiological or tissue effects, the safe implementation of a small oscillating volume modulation $(<1 \mathrm{ml})$ in even a large CSF space would be of concern.

\section{Conclusions}

This paper describes the initial studies of a cardiacgated volume-oscillating balloon suitable for intracranial implantation. These preliminary results suggest the ability to change the ICP waveform significantly in various ways, including augmentation and reduction of amplitude and phase shifts, without physiologically deleterious effects on mean ICP, systemic vascular effects, or brain injury. This device and technique may be used to study the role of ICP pulsatility in intracranial hemo- and hydrodynamic processes and introduces the more distant potential of cardiac-gated systems in various therapeutic applications involving blood flow and CSF solute distribution.

\section{Acknowledgments}

We would like to acknowledge Anthony Shawan (Mechanical
Prototype Core), Barry Kuban (Electronics Core), and Ji-Feng Chen (Polymer Core, Medical Device Solutions, Cleveland Clinic) for their help with the Cadence system and balloon construction, as well as Prashant Rawat, Tim Moran, and Jeff Arnold from CSF Therapeutics Inc., for engineering (P.R.) and administrative (T.M. and J.A.) help and consultation. This study was funded by an NIH grant awarded to Dr. Luciano (no. NS060916-01A2) and a Cleveland Clinic Innovations Grant for device development pilot testing also awarded to Dr. Luciano.

\section{Appendix}

The filtering of the ICP and laser Doppler blood flow signals consisted of fifth-order Butterworth filters with cutoffs at $0.5 \mathrm{~Hz}$ (high-pass) and $10 \mathrm{~Hz}$ (low-pass), implemented with MATLAB's built-in digital zero phase shift filtering algorithm. Filtered data were used to detect the time points of local extremes (i.e., peaks/ troughs) during the cardiac cycle, to calculate pulse amplitudes (maximum minus minimum), and for averaging of waveforms. Mean values and values at the local extremes were determined from unfiltered data.

A threshold-based peak detection algorithm for the R-wave of the EKG determined the start of each cardiac cycle. The start of balloon inflation (i.e., the achieved balloon cycle delay) was estimated as the point when the derivative of the balloon pressure first stayed below $-0.1 /$ above $+0.1 \mathrm{~mm} \mathrm{Hg} / \mathrm{msec}$ for 2 data samples $(-0.2 \mathrm{~mm} \mathrm{Hg} / \mathrm{msec}$ in 1 case $)$, after each $\mathrm{R}$-wave.

To perform ensemble averaging of ICP and the balloon pressure waveforms, the incremental data sets corresponding to each cardiac cycle were resampled to 100 time points, and the mean value for each time point was calculated over each baseline and activation segment. The mean pressure (unfiltered data) of the averaged cardiac cycles was then added to the resulting set of 100 mean values to create a waveform representative of the data segment. To avoid inconsistencies in the waveforms stemming from cardiac cycles where the balloon did not inflate properly, or from unusually long or short cardiac cycles, only cardiac cycles where the delay and pulse amplitude of the balloon cycle, as well as the cardiac cycle length, were within $\pm 10 \%$ of the median value were used. These criteria were only enforced to ensure the ensembleaveraged waveforms were illustrative of the typical effect of the different balloon waveforms, and were not applied to the numeric analysis of any of the ICP or CBF parameters. 


\section{References}

1. Bateman GA: Pulse-wave encephalopathy: a comparative study of the hydrodynamics of leukoaraiosis and normalpressure hydrocephalus. Neuroradiology 44:740-748, 2002

2. Bateman GA: Pulse wave encephalopathy: a spectrum hypothesis incorporating Alzheimer's disease, vascular dementia and normal pressure hydrocephalus. Med Hypotheses 62:182-187, 2004

3. Bilston LE, Stoodley MA, Fletcher DF: The influence of the relative timing of arterial and subarachnoid space pulse waves on spinal perivascular cerebrospinal fluid flow as a possible factor in syrinx development. J Neurosurg 112:808-813, 2010

4. Di Rocco C, Pettorossi VE, Caldarelli M, Mancinelli R, Velardi F: Communicating hydrocephalus induced by mechanically increased amplitude of the intraventricular cerebrospinal fluid pressure: experimental studies. Exp Neurol 59:40-52, 1978

5. Di Rocco C, Pettorossi VE, Caldarelli M, Mancinelli R, Velardi F: Experimental hydrocephalus following mechanical increment of intraventricular pulse pressure. Experientia 33:1470-1472, 1977

6. Egnor M, Zheng L, Rosiello A, Gutman F, Davis R: A model of pulsations in communicating hydrocephalus. Pediatr Neurosurg 36:281-303, 2002

7. Frank O: Die Grundform des arteriellen Pulses. Z Biol 37:483-526, 1899

8. Frank O: The basic shape of the arterial pulse. First treatise: mathematical analysis. 1899. J Mol Cell Cardiol 22:255277, 1990

9. Greitz D: Radiological assessment of hydrocephalus: new theories and implications for therapy. Neurosurg Rev 27:145-167, 2004

10. Greitz D, Wirestam R, Franck A, Nordell B, Thomsen C, Ståhlberg F: Pulsatile brain movement and associated hydrodynamics studied by magnetic resonance phase imaging. The Monro-Kellie doctrine revisited. Neuroradiology 34:370380, 1992

11. Iliff JJ, Wang M, Zeppenfeld DM, Venkataraman A, Plog BA, Liao Y, et al: Cerebral arterial pulsation drives paravascular CSF-interstitial fluid exchange in the murine brain. J Neurosci 33:18190-18199, 2013

12. Ji B, Ündar A: An evaluation of the benefits of pulsatile versus nonpulsatile perfusion during cardiopulmonary bypass procedures in pediatric and adult cardiac patients. ASAIO J 52:357-361, 2006

13. Kellie G: An account of the appearances observed in the dissection of two of three individuals presumed to have perished in the storm of the $3 \mathrm{~d}$, and whose bodies were discovered in the vicinity of Leith on the morning of the 4th, November 1821; with some reflection. Trans Med Chir Soc Edinburgh 1:84-169, 1824

14. Kress BT, Iliff JJ, Xia M, Wang M, Wei HS, Zeppenfeld D, et al: Impairment of paravascular clearance pathways in the aging brain. Ann Neurol 76:845-861, 2014

15. Laurent S, Katsahian S, Fassot C, Tropeano AI, Gautier I, Laloux B, et al: Aortic stiffness is an independent predictor of fatal stroke in essential hypertension. Stroke 34:12031206,2003

16. Luciano MG, Dombrowski SM, inventors; The Cleveland Clinic Foundation, assignee. Medical oscillating compliance devices and uses thereof. US patent US 8,956,379. February 17, 2015.

17. Luciano MG, Dombrowski SM, Moran T, inventors; The Cleveland Clinic Foundation, CSF Therapeutics, assignee. Device for increasing cerebral blood flow. US patent US 9,011,378. April 21, 2015.

18. Mitchell GF: Effects of central arterial aging on the structure and function of the peripheral vasculature: implications for end-organ damage. J Appl Physiol (1985) 105:1652-1660, 2008

19. Monro A: Observations on the Structure and Function of the Nervous System. Edinburgh: Creech and Johnson, 1783

20. Oldfield EH, Muraszko K, Shawker TH, Patronas NJ: Pathophysiology of syringomyelia associated with Chiari I malformation of the cerebellar tonsils. Implications for diagnosis and treatment. J Neurosurg 80:3-15, 1994

21. O'Rourke MF, Safar ME: Relationship between aortic stiffening and microvascular disease in brain and kidney: cause and logic of therapy. Hypertension 46:200-204, 2005

22. Pahlavian SH, Loth F, Luciano M, Oshinski J, Martin BA: Neural tissue motion impacts cerebrospinal fluid dynamics at the cervical medullary junction: a patient-specific movingboundary computational model. Ann Biomed Eng 43:29112923, 2015

23. Pettorossi VE, Di Rocco C, Mancinelli R, Caldarelli M, Velardi F: Communicating hydrocephalus induced by mechanically increased amplitude of the intraventricular cerebrospinal fluid pulse pressure: rationale and method. Exp Neurol 59:30-39, 1978

24. Ündar A: Myths and truths of pulsatile and nonpulsatile perfusion during acute and chronic cardiac support. Artif Organs 28:439-443, 2004

25. Wagshul ME, Eide PK, Madsen JR: The pulsating brain: A review of experimental and clinical studies of intracranial pulsatility. Fluids Barriers CNS 8:5, 2011

26. Wåhlin A, Ambarki K, Birgander R, Malm J, Eklund A: Intracranial pulsatility is associated with regional brain volume in elderly individuals. Neurobiol Aging 35:365-372, 2014

27. Weed LH, McKibben PS: Experimental alteration of brain bulk. Am J Physiol 48:531-558, 1919

28. Westerhof N, Lankhaar JW, Westerhof BE: The arterial Windkessel. Med Biol Eng Comput 47:131-141, 2009

\section{Disclosures}

Drs. Luciano and Dombrowski are listed as inventors on 2 patents regarding the device presented in this paper. This study received contractual research support from CSF Therapeutics Inc.

\section{Author Contributions}

Conception and design: Luciano, Dombrowski. Acquisition of data: Luciano, Dombrowski, El-Khoury, Yang. Analysis and interpretation of data: all authors. Drafting the article: Luciano, Qvarlander. Critically revising the article: Luciano, Dombrowski, Qvarlander, Thyagaraj, Loth. Reviewed submitted version of manuscript: all authors. Approved the final version of the manuscript on behalf of all authors: Luciano. Statistical analysis: Dombrowski, Qvarlander. Study supervision: Luciano.

\section{Supplemental Information}

\section{Previous Presentations}

Portions of this work were presented in abstract form at: 1) the 3rd Meeting of the International Society for Hydrocephalus and CSF Disorders, Baltimore, Maryland, September 2009; 2) the 54th Annual Meeting of the Society for Research into Hydrocephalus and Spina Bifida, Vancouver, Canada, July 8, 2010; 3) Neuroscience 2010 (Annual Meeting of the Society for Neuroscience), San Diego, California, November 3, 2010; and 4) the 1st International CSF Dynamics Symposium, Zurich, Switzerland, July 9, 2011.

\section{Correspondence}

Mark G. Luciano, Department of Neurosurgery, Johns Hopkins, 600 North Wolfe St., Phipps 126, Baltimore, MD 20287. email: mlucian4@jhmi.edu. 